\title{
The Estimation of the Mertens Function
}

\author{
Janusz Czopik \\ 2715 Cane Field Dr, Sugar Land, TX, USA \\ Email: jczopik@comcast.net
}

How to cite this paper: Czopik, J. (2019) The Estimation of the Mertens Function. Advances in Pure Mathematics, 9, 415-420. https://doi.org/10.4236/apm.2019.94019

Received: March 3, 2019

Accepted: April 26, 2019

Published: April 29, 2019

Copyright (c) 2019 by author(s) and Scientific Research Publishing Inc. This work is licensed under the Creative Commons Attribution International License (CC BY 4.0).

http://creativecommons.org/licenses/by/4.0/

\begin{abstract}
In this paper, we prove two formulas involving Mertens and Chebyshev functions. The first formula was done by Mertens himself without a proof. The second formula is a new one. Using these formulas, we estimate the Mertens function in such manner that we obtain a sufficient condition to approve the Riemann hypothesis.
\end{abstract}

\section{Keywords}

Mertens Function, Chebyshev Functions, Riemann Hypothesis

\section{Introduction}

The Mertens function $M(n)$ is defined as

$$
M(n)=\sum_{k \leq n} \mu(k)
$$

where $\mu(k)$ is the Möbius function. The function was named in honor of F.C.J. Mertens. Franz Carol Joseph Mertens was born on March 20th, 1840 in Sroda Prussia (now Środa Wielkopolska, Poland). He died on March 5th, 1927 in Vienna, Austria. The history of the attemps to proof the Riemann hypothesis started in 1885. Still in the records of the French Academy of Sciences, on July 13th, 1885, there is a note presented by Charles Hermite (the member of Academy) and written by a dutch mathematician Thomas Stjeltjes. He claims to have demonstrated the Riemann hypothese on one small page! The proof appeared false and Hermite explained why [1]. Stjeltjes never published the proof of the Riemann hypothesis. In 1897, Mertens based on empirical evidence claimed $|M(n)| \leq \sqrt{n}, n>1$ named for him the "Mertens conjecture" [2]. In 1985, Mertens conjecture was disproved by te Riele and Odlyzko using a high-speed computer [2]. There are several popular books about the Riemann hypothesis. For the list of those books, we can find in the Google [3]. In [1], we have the history of the zeta function of Riemann. Throughout this article, we will 
use letters: $k, n$ for natural numbers, $p$ for prime numbers and $x$ for real numbers, and also we assume $k \leq x$. The function $\log (x)=\log x=\log _{e}(x)=\ln x$. We will use Mertens function also for real numbers $x \geq 0 . M(x)$ is defined by

$$
\begin{gathered}
M(0)=0, \\
M([x])=\sum_{k \leq[x]} \mu(k) .
\end{gathered}
$$

The estimation of Mertens function is important for the number theory by the theorem proved in 1912 by J. E. Littlewood [4] (p. 261).

Theorem: The statement

$$
M(x)=O\left(x^{\frac{1}{2}+\epsilon}\right)
$$

for every $\epsilon>0$ is equivalent to the Riemann hypothesis.

\section{Some Properties of the Mertens Function}

First we recall formula [5]

$$
\sum_{k \leq x} M\left(\frac{x}{k}\right)=1 .
$$

Next, we give the new formula involving Mertens function and Chebychev function

$$
\psi(x)=\sum_{p^{m} \leq x} \log (p) .
$$

\section{Proposition 1.}

$$
\begin{gathered}
M(x) \log (x)=\sum_{k \leq x} \mu(k)\left(\log \left(\frac{x}{k}\right)-\psi\left(\frac{x}{k}\right)\right) . \\
\sum_{k \leq x} \mu(k)\left(\log \left(\frac{x}{k}\right)-\psi\left(\frac{x}{k}\right)\right)=\log (x) \sum_{k \leq x} \mu(k)-\sum_{k \leq x} \mu(k) \log (k) \\
-\sum_{k \leq x} \mu(k) \psi\left(\frac{x}{k}\right)=M(x) \log (x)-\sum_{k \leq x} \mu(k) \log (k)-\sum_{k \leq x} \mu(k) \psi\left(\frac{x}{k}\right) \\
=M(x) \log (x)
\end{gathered}
$$

because

$$
\sum_{k \leq x} \mu(k) \log (k)=-\sum_{k \leq x} \mu(k) \psi\left(\frac{x}{k}\right) .
$$

[6] (p. 107).

We shall prove the formula which was given by Mertens himself [7], without a proof, (proposition 2.)

Formula is of the form

Proposition 2.

$$
\psi(x)=\sum_{k \leq x} M\left(\frac{x}{k}\right) \log (k) .
$$

We state one of generalized Möbius inversion formulas [8] (p. 405) in the 
following form: let $f(x)$ be a function for $x \geq 1$ and

$$
g(x)=\sum_{k \leq x} f\left(\frac{x}{k}\right)
$$

Then for $x \geq 1$

$$
f(x)=\sum_{k \leq x} \mu(k) g\left(\frac{x}{k}\right),
$$

and reciprocally (vice versa).

Applying the Möbius formula as above to proposition 1 we get

$$
\sum_{k \leq x} M\left(\frac{x}{k}\right) \log \left(\frac{x}{k}\right)=\log (x)-\psi(x) .
$$

On the other hand we have

$$
\begin{gathered}
\sum_{k \leq x} M\left(\frac{x}{k}\right) \log \left(\frac{x}{k}\right)=\sum_{k \leq x} M\left(\frac{x}{k}\right) \log (x)-\sum_{k \leq x} M\left(\frac{x}{k}\right) \log (k) \\
=\log (x) \sum_{k \leq x} M\left(\frac{x}{k}\right)-\sum_{k \leq x} M\left(\frac{x}{k}\right) \log (k) \\
=\log (x)-\sum_{k \leq x} M\left(\frac{x}{k}\right) \log (k) .
\end{gathered}
$$

Finally we have

$$
\log (x)-\psi(x)=\log (x)-\sum_{k \leq x} M\left(\frac{x}{k}\right) \log (k),
$$

so

$$
\psi(x)=\sum_{k \leq x} M\left(\frac{x}{k}\right) \log (k) .
$$

This completes the proof.

Notice. The formulas used in the paper are some kind of identities. They follow from the properties of Mertens and Chebyshev functions.

\section{The Estimation of Mertens Function}

From proposition 1 we have

$$
\begin{aligned}
M(x) \log (x) & =\sum_{k \leq x} \mu(k) \log \left(\frac{x}{k}\right)-\sum_{k \leq x} \mu(k) \psi\left(\frac{x}{k}\right) \\
|M(x) \log (x)| & =\left|\sum_{k \leq x} \mu(k) \log \left(\frac{x}{k}\right)-\sum_{k \leq x} \mu(k) \psi\left(\frac{x}{k}\right)\right| \\
= & \left|\sum_{k \leq x} \mu(k)\left(\log \left(\frac{x}{k}\right)-\psi\left(\frac{x}{k}\right)\right)\right| \\
& \leq \sum_{k \leq x}\left|\log \left(\frac{x}{k}\right)-\psi\left(\frac{x}{k}\right)\right| .
\end{aligned}
$$

Because $\log x \leq \theta(x)$ for all $x \geq 1$, where

$$
\theta(x)=\sum_{p \leq x} \log (p)
$$


we replaced $\log \left(\frac{x}{k}\right)$ by something greater, i.e. by $\theta\left(\frac{x}{k}\right)$ and we get

$$
\sum_{k \leq x}\left|\log \left(\frac{x}{k}\right)-\psi\left(\frac{x}{k}\right)\right| \leq \sum_{k \leq x}\left|\theta\left(\frac{x}{k}\right)-\psi\left(\frac{x}{k}\right)\right| .
$$

We have

$$
\psi(x)=\sum_{m=1}^{\infty} \theta\left(x^{1 / m}\right)
$$

for all $x \geq 1$, [8] p. 318,

and

$$
\psi\left(\frac{x}{k}\right)=\sum_{m=1}^{\infty} \theta\left(\left(\frac{x}{k}\right)^{1 / m}\right) .
$$

Notice. We use the symbol of sigma from 1 to infinity but the number of summand different from zero is always finite.

Next note that on the right hand side of above formula if $\left(\frac{x}{k}\right)^{1 / m}<2$ then the corresponding summands

$$
\theta\left(\left(\frac{x}{k}\right)^{1 / m}\right)=0 .
$$

Let

$$
\begin{aligned}
& m=\left(\log \left(\frac{x}{k}\right)\right) / \log (2)=(\log (x)-\log (k)) / \log (2)=\frac{\log (x)}{\log (2)}-\frac{\log (k)}{\log (2)} . \\
& \text { If } m>\frac{\log x}{\log 2}
\end{aligned}
$$

then

$$
\begin{gathered}
\theta\left(\left(\frac{x}{k}\right)^{1 / m}\right)=0 . \\
\psi\left(\frac{x}{k}\right)=\sum_{m=1}^{\infty} \theta\left(\left(\frac{x}{k}\right)^{1 / m}\right) . \\
\psi\left(\frac{x}{k}\right)-\theta\left(\frac{x}{k}\right)=\sum_{m=2}^{\infty} \theta\left(\left(\frac{x}{k}\right)^{1 / m}\right) .
\end{gathered}
$$

We know [8], p. 319

$$
\theta(x)=O(x \log (x))
$$

and

$$
\begin{gathered}
\psi\left(\frac{x}{k}\right)-\theta\left(\frac{x}{k}\right)=\sum_{m=2}^{\infty} O\left(\left(\frac{x}{k}\right)^{1 / m} \log \left(\frac{x}{k}\right)\right) \\
=O\left(\left(\frac{x}{k}\right)^{1 / 2} \log \left(\frac{x}{k}\right)\right)+\sum_{3 \leq m \leq \log (x) / \log (2)} O\left(\left(\frac{x}{k}\right)^{1 / 3} \log ^{2}\left(\frac{x}{k}\right)\right)
\end{gathered}
$$




$$
=O\left(\left(\frac{x}{k}\right)^{1 / 2} \log \left(\frac{x}{k}\right)\right) .
$$

(There are at most $\log \left(\frac{x}{k}\right)$ nonzero terms in last sum).

\section{Results}

Finally, according to

$$
\begin{gathered}
|M(x) \log (x)| \\
\leq \sum_{k \leq x}\left|\theta\left(\frac{x}{k}\right)-\psi\left(\frac{x}{k}\right)\right|=\sum_{k \leq x}\left(\psi\left(\frac{x}{k}\right)-\theta\left(\frac{x}{k}\right)\right) \\
=\sum_{k \leq x} O\left(\left(\frac{x}{k}\right)^{1 / 2} \log \left(\frac{x}{k}\right)\right)=O\left(x^{1 / 2} \log (x)\right),
\end{gathered}
$$

we obtain

$$
|M(x)| \log (x)=O\left(x^{1 / 2} \log (x)\right) .
$$

From the definition of big " $O$ " notation we have $|M(x)| \log (x) \leq K x^{\frac{1}{2}} \log (x)$ for all $x \geq 1$ where $K>0$.

Thus $|M(x)| \leq K x^{\frac{1}{2}}, \quad x \geq 1$ i.e.

$$
M(x)=O\left(x^{\frac{1}{2}}\right) .
$$

The result

$$
M(x)=O\left(x^{\frac{1}{2}}\right)
$$

is the sufficient condition for the approval of Riemann hypothesis.

In [4] chapter 12.1, we can find more about that.

\section{Conclusion}

The estimation of the Mertens function $M(x)$ is in the form as in theorem of the Lttlewood [4]. The result means the Riemann hypothesis is the theorem. The future problem is to find an exact formula for an imaginary part of the zeros of the Riemann zeta function.

\section{Conflict of Interest}

The author declares no conflict of interest regarding the publication of this paper.

\section{References}

[1] http://fracademic.com/dic.nsf/frwiki/783000

[2] Odlyzko, A.M. and te Riele, H. (1985) Disproof of Mertens Conjecture. Journal für 
die reine und angewandte Mathematik, 357, 138-160.

[3] http://riemannhypothesis.info/books/books-on-the-riemann-hypothesis/

[4] Edwards, H.M. (2001) Riemann's Zeta Function. Dover Publication, Mineola, New York.

[5] http://mathworld.wolfram.com/MertensFunction.htlm

[6] Grosswald, E. (1966) Topics from the Theory of Numbers. The Macmillan Company, University of Pennsylvania.

[7] http://en.wikipedia.org/wiki/Mertens-conjecture

[8] Shapiro, H.N. (2008) Introduction to the Theory of Number. Dover, Publications, Mineola. https://doi.org/10.1353/tae.2008.0008 